\title{
A Gas-Kinetic Scheme for Turbulent Flow
}

\author{
Marcello Righi ${ }^{1}$
}

Received: 5 January 2015 / Accepted: 19 October 2015 / Published online: 26 November 2015

(C) Springer Science+Business Media Dordrecht 2015

\begin{abstract}
RANS simulations may not provide accurate results for all flow conditions. The interaction between a shock wave and a turbulent boundary layer is an example which may still be difficult to simulate accurately. Beside the inability to reproduce physical phenomena such as shock unsteadiness, the argument is put forward that the conventional numerical schemes, based on the Navier-Stokes equations, may be unable to generate a physically consistent turbulent stress tensor in the presence of large unresolved scales of motion. A large ratio between unresolved and resolved scales of motion, a sort of Knudsen number based on turbulent fluctuations, might introduce inaccuracies for which the turbulence model is not accountable. In order to improve the accuracy of RANS simulations, researchers have suggested various ad-hoc modifications to standard turbulence models which limit eddy viscosity or the turbulent stress tensor in the presence of strong gradients. Gas-kinetic schemes might be able to improve RANS predictions in shocklayers by removing or limiting the errors caused by the large scales ratio. These schemes are a class of their own; in the framework of a finite-volume or finite-elements discretizations, they model the numerical fluxes on the basis of the Boltzmann equation instead of the Navier-Stokes equations as is conventionally done. In practical terms, these schemes provide a higher accuracy and, more importantly, an in-built "multiscalar" mechanism, i.e. the ability to adjust to the size of unresolved scales of motion. This property makes them suitable for shock-capturing and rarefied flow. Gas-kinetic scheme may be coupled to a conventional RANS turbulence model; it is shown that the turbulent stress tensor is naturally adjusted as a function of the unresolved-toresolved scales ratios and achieves a higher physical consistency than conventional schemes. The simulations shown - well-known benchmark cases with strong shock-boundary layer interactions - have been obtained with a standard two-equation turbulence model $(k-\omega)$. It is shown that the gas-kinetic scheme provides good quality predictions, where conventional schemes with the same turbulence model are known to fail.
\end{abstract}

Marcello Righi

rigm@zhaw.ch

1 Zurich University of Applied Sciences, Technikumstrasse 9, 8401 Winterthur, Switzerland 
Keywords Gas kinetic theory · Turbulence modeling · Compressible flow • Shock boundary layer interaction

\section{Introduction}

Modeling the unresolved turbulent scales of motion was inspired by the diffusion process which models thermal fluctuations in the continuum approach to fluid mechanics. However, unlike thermal fluctuations, unresolved turbulent fluctuations are spread over a range of frequencies / wavenumbers and a clear separation from the resolved scales of motion may not exist. In LES, a cut-off wavenumber in the inertial range may even be a pre-requisite. Interestingly, when considering the numerical simulation of rarefied flow, the ratio of the largest unresolved scale of motion to the smallest (or a representative) resolved one - known as Knudsen number - is precisely the indicator of the degree of rarefaction and the approaching of the validity limit of the conventional Navier-Stokes approach. Large unresolved scales motion may invalidate the splitting, on mathematical and numerical level, of advective and diffusive fluxes. This is indeed well accepted when dealing with rarefied flow; as the Knudsen number approaches a given limit, normally between 0.001 and 0.01 , researchers and engineers know that prediction methods based on the continuum model, such as those derived from the Euler or Navier-Stokes equations, may become inaccurate and unreliable. Typically, approaches such as discrete velocity methods [25, 37, 49], the Direct Simulation Monte Carlo (DSMC) [4, 6] or Molecular Dynamics [1, 27] are employed (refer to [7, 42] and references therein for an extensive discussion).

In turbulent simulations, one might want to introduce the measure of a virtual degree of rarefaction given by the ratio of unresolved turbulent scales of motion to a reference resolved scale. The use of the expression "rarefaction" has not to be considered literally. In the RANS approach, the effect of all turbulent scales of motion, including the large ones, are accounted for by a modeled stress tensor. The (turbulent) unresolved-to-resolved scales ratio assumes large values in some flow regions, such as shocklayers, due to presence of strong (resolved) gradients and the amplification of turbulence intensity caused by compression shocks. In this paper (Section 3), this ratio is assessed in a few cases and shown to reach peak values of several hundredths or even a few tenths; i.e. well beyond the threshold of rarefaction.

RANS simulations of these regions are indeed challenging: it is well accepted that numerical simulations based on the RANS approach (also LES or mixed methods), may still prove very challenging as the prediction of both turbulent quantities and turbulent stress tensor may require special or ad-hoc modeling techniques. Typically, numerical simulations may fail to accurately capture flow details such as strength and position of the shock, position of separation and re-attachment, turbulence intensity downstream of the separated flow region and in particular heat flux in the case of high enthalpy flows. The turbulence model is invariably the culprit. In practice, this inability to reach engineering standard quality, may negatively affect the design of supersonic or hypersonic vehicles, leading to unsuitable geometries or high development costs. Refer to [2] and references therein for a comprehensive discussion.

In order to improve the RANS prediction of turbulent shocklayers, the efforts of researchers and engineers focus largely on turbulence modeling, by either improving the evolution of turbulent quantities [23, 41] and/or limiting [41] / improving [17, 38] the turbulent stress tensor. Refer to [24] for transonic flow and [30] for hypersonic flows. This study addresses instead the role of the numerical scheme in the prediction of such flows. In particular, a gas-kinetic scheme is used to run RANS simulations. Turbulence modeling is 
obtained with a standard two-equation turbulence model, the $k-\omega$ in the original formulation [40], i.e. without the correction terms proposed by Wilcox in 2008 [41]. This particular model has been chosen precisely because it is known to overestimate turbulent stresses in the presence of separation or incipient separation [38, 41]. The strength of the gas-kinetic schemes lies in the ability to evaluate advective and diffusive fluxes in a single operation, including therefore the effect of "collisions" on "transport", or, in other words, to take into account the scale of the unresolved fluctuations - introducing a "multiscale" effect which is missing in Navier-Stokes schemes. This is evident in the derivation of the scheme (Section 2) where it is shown that the scheme adjusts itself as a function of the unresolved-to-resolved scales ratio, limiting diffusion in the presence of large unresolved fluctuations. Interestingly, conventional turbulence modeling also uses the ratio of turbulent to resolved lengthscales to introduce second order effects $[17,38,41]$. Further remarks concern the fact that (i) the gas-kinetic scheme uses the timescales instead of the (usual for turbulence modeling) lenghtscales; and (ii) the inclusion of these kinetic effects also generates a misalignment between turbulent stress and strain rate acting in a similar way to high-order turbulence models. This is discussed in $[8,9]$.

Gas-kinetic schemes are derived form the Boltzmann equation and not from the NavierStokes or Euler equations. They are suitable for finite volume or finite elements schemes. Being relatively little known, they are often confused with particle methods such as DSMC or Molecular Dynamics or with Lattice Boltzmann methods. Numerous gas-kinetic schemes have been developed over the latest twenty years [10, 21, 22, 43, 47] with the aim to achieve a physically more consistent mathematical model of fluid mechanics. Gas-kinetic schemes are more accurate than conventional schemes, and might be able to resolve shock-layers. Besides, they are more suitable to high-order reconstruction $[19,46,48]$ and may be used as a platform to investigate rarefied flow [20,45]. Since conservation laws such as the NavierStokes and Euler equations can be derived from the Boltzmann equation, as shown in [6, 42], a gas-kinetic scheme is always consistent with a conventional one, within the validity boundaries of the latter. To build the turbulent gas-kinetic scheme shown in this paper, the scheme developed by $\mathrm{Xu}$ in 2001 [43] has been the starting point.

The derivation of the turbulent gas-kinetic scheme is presented in Section 2, the implementation of the scheme into a finite-volume solver the results and the results of the numerical experiments are summarized in Section 3. Conclusions are presented in Section 4.

\section{Gas-Kinetic Schemes}

\subsection{A gas-kinetic scheme for laminar flow}

The state of a gas can be described by means of a distribution function $f(x, v, t)$ - defined in the phase space. The conservative variables $w=\left[\begin{array}{lllll}\rho & \rho v_{1} & \rho v_{2} & \rho v_{3} & \rho E\end{array}\right]^{T}$ are recovered from $f$ by taking moments in the phase space:

$$
w=\int \psi f d \Xi
$$

where the elementary volume in phase space is $d \Xi=d u_{1} d u_{2} d u_{3} d \xi$ and:

$$
\psi=\left[\begin{array}{lllll}
1 & u_{1} & u_{2} & u_{3} & \frac{1}{2}\left(u_{i}{ }^{2}+\xi^{2}\right)
\end{array}\right]^{T} \text {. }
$$


The numerical fluxes $\mathcal{F}$ related to a unit interface length normal to direction $n$, and a time step $\Delta t$ are obtained by time integration:

$$
\mathcal{F}_{n}=\int_{0}^{\Delta t} \int f \psi u_{n} d \Xi d t
$$

The distribution function $f$ is assumed to be a solution of the Boltzmann-BGK equation [3]:

$$
\frac{\partial f}{\partial t}+(u \cdot \nabla) f=\frac{f^{e q}-f}{\tau},
$$

where $\tau$ is a relaxation time corresponding to the "average" fluctuation period and proportional to molecular viscosity divided by static pressure in laminar flow:

$$
\tau=\frac{\mu}{p}
$$

$f^{e q}$ is a Maxwellian distribution, representing a fluid in thermal equilibrium:

$$
f^{e q}=\rho\left(\frac{\lambda}{\pi}\right)^{\frac{N+2}{N}} \exp \left[-\lambda\left(\left(u_{i}-v_{i}\right)^{2}+\xi^{2}\right)\right],
$$

where $\lambda=\rho / 2 p=1 / 2 T$ and $N$ is the number of effective degrees of freedom of the molecules. Refer to $[10,42,43]$ for additional details. It is evident that Eq. 4 includes the transport terms and a relaxation process towards thermal equilibrium. The latter models the effect of molecular collisions, i.e. viscosity in the continuum regime. A solution to Eq. 4 is sought in terms of a series expansion of $f$ around a Maxwellian distribution: the Chapman-Enskog expansion $[6,42]$ is used to model the deviations from the thermal equilibrium. Various expansion orders may be used in the derivation of a gas-kinetic scheme. A Chapman-Enskog expansion to the zeroth-order, i.e.:

$$
f=f^{e q},
$$

ignores the thermal fluctuations and is equivalent to the Euler representation of a gas (this can be proven by inserting (7) into Eq. 4 and taking the moment as in Eq. 1). A first-order expansion includes a linear "collisions" term:

$$
f=f^{e q}-\epsilon \widehat{\tau} D f^{e q}+\ldots
$$

where $\widehat{\tau}$ is a reference relaxation time and $\epsilon$ is a "small" number, which represents a measure of the deviations from thermal equilibrium. As is well known, Eq. 4 with a first-order Chapman-Enskog expansion allows the derivation of the Navier-Stokes equations [6, 42].

A Chapman-Enskog expansion to the second-order is expressed as:

$$
f=f^{e q}-\epsilon \widehat{\tau} D f^{e q}+\epsilon^{2} \widehat{\tau} D\left(\widehat{\tau} D f^{e q}\right)+\ldots
$$

Inserting Eq. 9 into Eq. 4 would allow the recovery of the Burnett equations [6]. The third truncation order, not shown here, provides the Super Burnett set of equations. Despite the fact that these higher-order expansions provide a more accurate model of unresolved fluctuations, no convincing applications to rarefied have been put forward so far (refer to the discussion in [6]). However, higher-order expansions are being used with gas-kinetic schemes $[19,26]$ with the aim of achieving a higher accuracy and handling local rarefaction.

This study relies on a first-order expansion. However, a modification is introduced in order to partially re-instate second-order accuracy in locally rarefied flow. This is presented in Section 2.2.

The scheme derived by $\mathrm{Xu}$ [43] uses the monotone upstream-centered scheme for conservation laws (MUSCL) approach and assumes a discontinuous reconstruction. The gas 
evolution is integrated in time at each time step. An initial distribution $f_{0}$ is generated on the basis of the gas states and adopting a second-order Chapman-Enskog expansion. Further, a distribution function $f^{B G K}$ is obtained as the analytical solution to the BGK equation, Eq. 4:

$$
\begin{array}{rl}
f & B G K \\
& \left(x_{1}, x_{2}, x_{3}, t, u_{1}, u_{2}, u_{3}, \xi\right) \\
= & \frac{1}{\tau} \int_{o}^{t} f^{e q}\left(x_{1}^{\prime}, x_{2}^{\prime}, x_{3}^{\prime}, t, u_{1}, u_{2}, u_{3}, \xi\right) e^{-\left(t-t^{\prime}\right) / \tau} d t^{\prime} \\
& \quad+e^{-t / \tau} f_{0}\left(x_{1}-u_{1} t, x_{2}-u_{2} t, x_{3}-u_{3} t\right),
\end{array}
$$

where $x_{1}^{\prime}=x_{1}-u_{1}\left(t-t^{\prime}\right), \quad x_{2}^{\prime}=x_{2}-u_{2}\left(t-t^{\prime}\right), \quad x_{3}^{\prime}=x_{3}-u_{3}\left(t-t^{\prime}\right)$. The initial distribution $f_{0}$ includes spatial and temporal gradients calculated from the macroscopic variables. The details of the derivation can be obtained in $[28,29,43]$. In this paper, the resulting distribution function, solution to the BGK model and compatible with the gas initial states, $f^{B G K}$, is expressed in a compact form

$$
f^{B G K}=f^{N S}+e^{-t / \tau} \Delta f
$$

where $f^{N S}$ is a first-order Chapman-Enskog expansion built around an average gas state at the interface, whereas $\Delta f$ is a more complex function, obtained as difference between $f^{N S}$ and a Chapman-Enskog expansion obtained combining the "left" and "right" gas states. The presence of the blending function $e^{-t / \tau}$ is important and represents the kernel of the "multiscalar" mechanism of the gas-kinetic scheme. It is important to remark that $f^{N S}$ generates fluxes which might be compared to the ones provided by a central conventional scheme. The fluxes are finally obtained inserting $f=f^{B G K}$ into Eq. 3; they can be expressed as:

$$
\mathcal{F}_{n}=\mathcal{F}_{n}^{N S}+\alpha(\epsilon) \mathcal{F}_{n}^{\Delta},
$$

where $\mathcal{F}_{n}^{N S}$ are the fluxes associated with $f^{N S}$ and $\mathcal{F}_{n}^{\Delta}$ the ones associated with $\Delta f$, $\left.\alpha(\epsilon)=\epsilon\left(1-e^{-1 / \epsilon}\right)\right)$ and $\epsilon=\tau / \Delta t$. At first sight, (12), bears some resemblance with some upwind schemes: central fluxes are corrected by additional terms, whose role becomes more important the stronger the gradients involved. However, in Eq. 12 the parameter $\epsilon$ plays an important role too. $\epsilon=\tau / \Delta t$ is the ratio between two time scales: $\tau$ relates to the relaxation process and hence to diffusion in physical space: it can be considered as a representative time scale of the unresolved scales of motion. $\Delta t$, the time step length, is related to the smallest resolved time scale. $\epsilon$ is therefore the ratio of unresolved to resolved time scales. The "multiscale" mechanism in gas-kinetic schemes is evident: the higher is $\epsilon$, the larger are the corrections to the Navier-stokes fluxes. This mechanism alone does not suffice for a gas dynamics scheme to provide a physically consistent model of rarefied gas. The relaxation time must also provide a consistent model of particle collisions. As far as laminar flow is concerned, a few studies have demonstrated the suitability of this gas-kinetic scheme to moderate rarefaction $[20,44]$. This study proposes a solution for turbulent flow in 2.2.

The dissipative mechanism in this type of gas-kinetic scheme has been thoroughly investigated by $\mathrm{Xu}$ [43]: whereas in smooth flow dissipation it is equivalent to the diffusion process in the Navier-Stokes equations, in the presence of discontinuities in the variables reconstruction, the scheme introduces kinetic terms originating from the initial conditions ( $f_{0}$ in Eq. 10) but does not add any artificial dissipation. In order to provide numerical stability across shocks which cannot be resolved some artificial dissipation is explicitly 
introduced. This is achieved by adding to the relaxation time a term proportional to the time step length and the pressure jump across the interface [43]:

$$
\tau=\frac{\mu}{p}+C \Delta t \frac{\left(p^{l}-p^{r}\right)}{\left(p^{l}+p^{r}\right)},
$$

where $p^{l}$ and $p^{r}$ are the reconstructed pressure values at the interface, $\Delta t$ is the time step length, $C$ is a coefficient, which can be adjusted empirically and is always in the order of the unity. In this study, the choice of $C$ has never been instrumental in achieving accurate predictions of the shock - boundary layer interaction. In most cases the shocklayers investigated spread across several computational grids; this makes the role of the artificial dissipation less significant.

As a final remark, the BGK model is consistent with conservation laws with an unity Prandtl number; the heath flux must therefore be corrected for realistic fluids [43]. A number of publications $([19,46,48]$ and references therein) demonstrate the good qualities of the scheme.

\subsection{A gas-kinetic scheme for turbulent flow}

Assuming that the effect of unresolved turbulent scales of motion can be represented by a diffusion process and that eddy viscosity $\mu_{t}$ is available, the relaxation time to use in Eq. 4 can be trivially set to:

$$
\tau=\frac{\mu+\mu_{t}}{p} .
$$

The time scale ratio $\epsilon$ which drives the correction terms in the numerical fluxes would therefore become:

$$
\epsilon=\frac{\tau}{\Delta t}=\frac{\mu+\mu_{t}}{p \Delta t} .
$$

It is evident that in particular flow regions, such as shocklayers, Eq. 15 may drive $\epsilon$ to rather large values, as it was pointed out in the Introduction section to this paper. Large values of $\epsilon$ imply "mathematical" rarefaction, which does not reflect in the physical rarefaction of the flow, but still affect the physical consistence of the approach questionable. The idea of eddy viscosity was born from the analogy between a distribution of particles and a distribution of eddies in a turbulent flow. The rarefaction highlighted by Eq. 15 would imply the lack of a clear separation between the scale of motion of the eddies and of the resolved or mean flow.

Since the gas-kinetic scheme also allows considering moderate rarefaction, this aspect of RANS simulations may also be taken into account. Xu [44] has proposed the following approach for moderately rarefied (laminar) flow; following [44]: a first-order expansion is used as in Eq. 8, whereas the relaxation time $\tau$ is adjusted in order to locally achieve secondorder accuracy, as in Eq. 9. A generalised relaxation time $\tau^{*}$, which becomes a function of local gradients, can be enforced in the Chapman-Enskog expansion, Eq. 8:

$$
f=f^{e q}-\tau^{*} D f^{e q},
$$

which is then inserted into the BGK equation (4) in order to obtain the dependence of $\tau^{*}$ on the flow gradients:

$$
\tau^{*}=\frac{\tau\left(1-D \tau^{*}\right)}{1+\tau\left(D^{2} f^{e q} / D f^{e q}\right)} .
$$


As pointed out in [44], the term $\tau D^{2} f^{e q} / D f^{e q}$ can be considered as a lengthscale ratio, i.e. proportional to the Knudsen number in the continuum regime. To the leading order, Eq. 17 can be reduced to:

$$
\tau^{*} \simeq \frac{\tau}{1+\tau\left(D^{2} f^{e q} / D f^{e q}\right)} .
$$

Interestingly enough, a qualitatively similar result has been obtained in $[8,9]$ from different considerations:

$$
\tau=\frac{\mu}{p}+\frac{\mu_{t}}{\rho T\left(1+\eta^{2}\right)^{1 / 2}},
$$

where $T$ is temperature and $\eta=S k / \varepsilon=S / \omega$ is a time scales ratio, $S$ is a measure of the local velocity gradient. $k, \varepsilon$ and $\omega$ are the well known turbulent kinetic energy, dissipation and specific dissipation. Molecular viscosity has been added separately. Note that considering the leading order and same sign derivatives, Eqs. 18 and 19 (considering only the turbulent part) both adjust the relaxation time as a function of a scale ratio $\epsilon$ :

$$
\tau^{*}=\tau \frac{1}{1+\epsilon} \text {. }
$$

In this study, Eq. 19 has been preferred to Eq. 18 in order to avoid the calculation of gradients. It is worthwhile mentioning that an unresolved-to-resolved scales ratio, typically a term proportional to $\eta=S / \omega$, is used to adjust or limit the expression of eddy viscosity, e.g. in the limiting value for $\omega$ proposed by Wilcox [41].

\subsection{Modification to the scheme to introduce a more robust time scale ratio}

In turbulent flow, the quantity $\epsilon=\tau / \Delta t$ may assume large values, depending on the time advancing technique and grid, leading to a lack of robustness and grid dependent results. It is proposed to replace it with:

$$
\epsilon=\frac{\tau}{\widetilde{\tau}},
$$

where $\tilde{\tau}$ is the assumed timescale of the resolved flow and can be calculated form the gradients of one of the resolved variables (i.e. $\tilde{\tau}=\rho / D \rho$ ). Details are explained in [29]. The expression of $\epsilon$ used in Eq. 21 is close to the one used in rarefied gas dynamics to estimate the local Knudsen number, with the difference that in this case timescales are used instead of spatial quantities. In the section dedicated to the results obtained, it can be seen that this timescale ratio or "degree of rarefaction" may assume values up to a few hundredths inside shocklayers. In rarefied gas dynamics the validity of Navier-Stokes schemes may become questionable for Knudsen number as high as one thousandth. The use of a gaskinetic scheme with eddy viscosity may therefore exploit its better ability to cope with these "rarefaction" levels.

\subsection{Second-order turbulent stress tensor obtained from the second-order Chapman-Enskog expansion}

It has been demonstrated by Chen in [9] that a gas-kinetic scheme built around a secondorder Chapman-Enskog expansion ( 8 truncated to the second order) would generate a nonlinear turbulent stress tensor $R_{i j}=-\frac{2}{3} \rho k \delta_{i j}+2 \mu_{t} S_{i j}+\mu_{t}^{2} /(\rho k) R_{i j}^{(2)}$. The second order term, $R_{i j}^{(2)}$, can be expressed as a function of the velocity fields $u_{i}$ as:

$$
R_{i j}^{(2)}=\left[C_{1} \frac{\partial u_{i}}{\partial x_{k}} \frac{\partial u_{j}}{\partial x_{k}}+C_{2}\left(\frac{\partial u_{i}}{\partial x_{k}} \frac{\partial u_{k}}{\partial x_{j}}+\frac{\partial u_{j}}{\partial x_{k}} \frac{\partial u_{k}}{\partial x_{i}}\right)+C_{3} \frac{\partial u_{k}}{\partial x_{i}} \frac{\partial u_{k}}{\partial x_{j}}\right] .
$$


Chen et al. [9] have demonstrated that the second order Chapman-Enskog expansion leads to values for the numerical $C_{1}, C_{2}$ and $C_{3}$ surprisingly close to those selected by turbulence researchers based on empirical methods $[31,36,50]$. This clearly represents a promising way forward, which has not yet been implemented into a gas-kinetic scheme.

\subsection{Kinetic wall boundary condition}

The idea of a kinetic wall boundary condition has been adapted from [10, 18, 44]. The fluxes are calculated by modelling the gas evolution stage within the gas-kinetic solver starting from a "wall" state and a "flow" state. The former is set at wall conditions, i.e. at wall temperature, whereas the latter is extrapolated from inside the computational domain. The gas evolution is modelled assuming that fluctuations from the flow domain towards the wall are at "flow" conditions whereas the ones in the opposite directions are at "wall" conditions. The distribution function $f$ considered to assess the fluxes between wall and flow domain is:

$$
f=(1-\beta) f_{u_{1}>0}^{W}+\beta f_{u_{1}>0}^{F}+f_{u_{1}<0}^{F},
$$

where $f^{W}$ and $f^{F}$ are "wall" and "flow" distribution respectively. The velocity $u_{1}$ is normal to the wall and positive outwards. The parameter $\beta$ provides the indication of how much the flow has accommodated to wall conditions, i.e. $\beta=0$ implies full accommodation. Slip velocity is automatically generated since the velocity parallel to the wall calculated using inserting (23) into Eq. 1 may not be zero:

$$
v_{2}=\int u_{2} f d \Xi
$$

\section{Numerical Experiments}

\subsection{Numerical implementation}

The gas-kinetic scheme described above has been implemented into a two-dimensional finite-volume steady-state solver. The simulations of transonic and supersonic flows have been run with a second-order reconstruction and the minmod limiter for the reconstruction
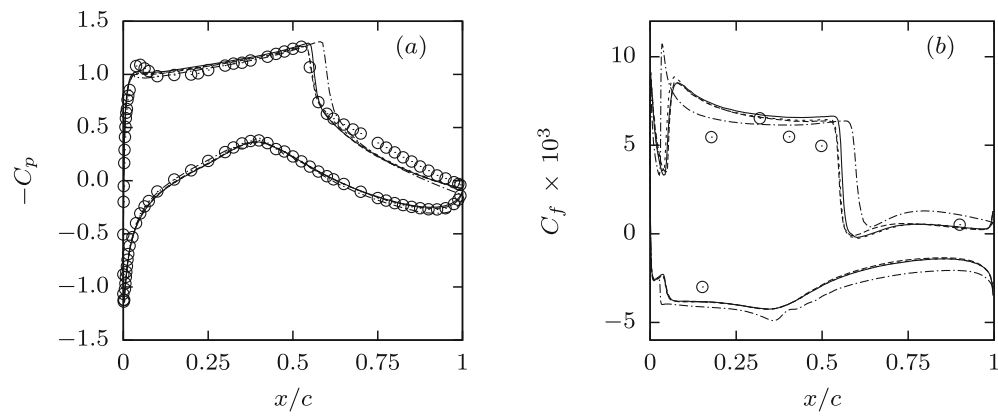

Fig. 1 RAE2822 airfoil (Case $10 R e=6.2 \times 10^{6}, M=0.745$, angle of attack $\left.\alpha=3.19^{\circ}\right)$. ( $(-)$ Gas-kinetic scheme (GKS) on finest grid, ( - - _ -) GKS on medium grid, ( - . - . -) GKS on coarsest grid, $(-\cdot-)$ Navier-Stokes (Roe's approximate Riemann solver) on finest grid, ( $\circ$ ): experimental data from Cook [12]. a pressure coefficient, b skin friction coefficient. Fine grid size: $560 \times 176$ 
of conservative variables and their gradients. Hypersonic flows have been simulated with a WENO 5 reconstruction. The relaxation time has been calculated with Eq. 14, where the eddy viscosity is obtained from a linear $k-\omega$ model:

$$
\tau=\frac{\mu}{p}+\frac{k / \omega}{T\left(1+\eta^{2}\right)^{1 / 2}} .
$$

The solver uses a LU-SGS preconditioning and runs with CFL between 4 and 20, depending on grid and flow. The overall computational cost requested by the gas-kinetic scheme is higher by a factor of two on average. However, the solver is sequential and no attempts have been done to optimize the code for speed. No wall functions have been used, the viscous sublayer has been accurately resolved in every flow case. Hypersonic cases have been run with kinetic wall conditions.

A grid convergence exercise has been carried out for each flow case type. In this study no grid adaptation has been used; finer grids have been obtained from uniform refinement. However, $\mathrm{Xu}$ has shown [43] that adaptive refinement inside a shocklayer allows a gaskinetic scheme to calculate a physical consistent solution of the shock.

\subsection{Time scales ratio}

In this section results of transonic, supersonic and hypersonic flows are shown. The unresolved-to-resolved timescales ratio, calculated on the basis of Eq. 21, is shown for some
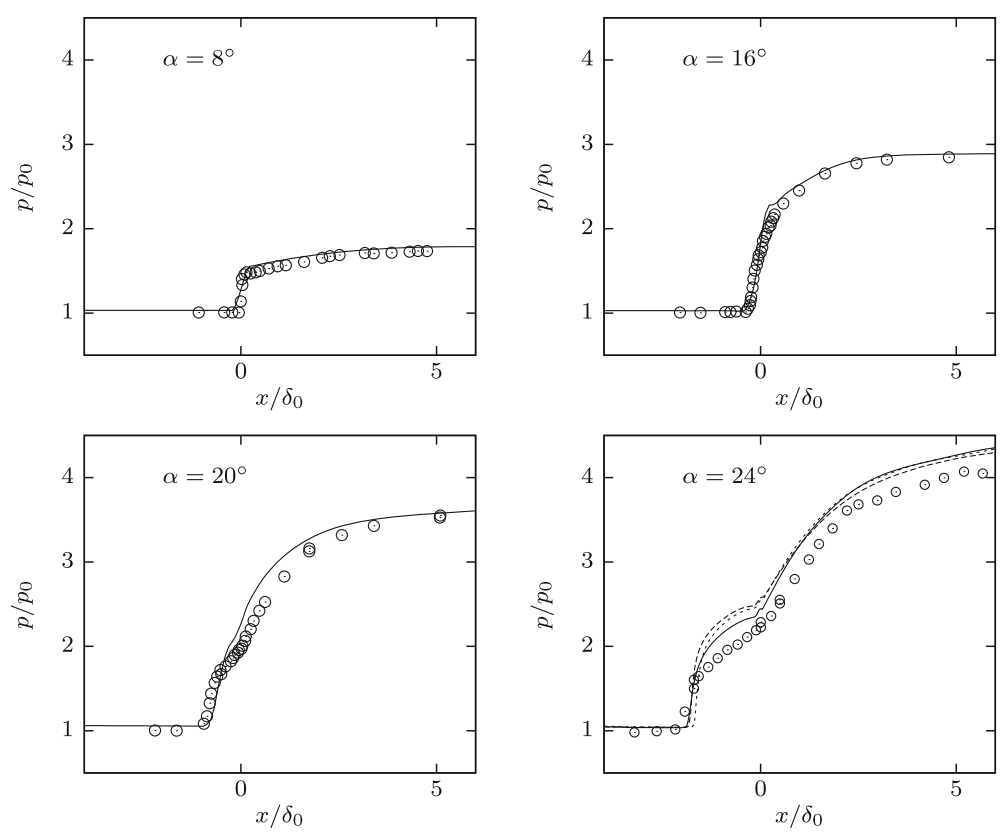

Fig. 2 Pressure calculated for four different compression corner flows, characterized by angles values of $8^{\circ}, 16^{\circ}, 20^{\circ}$ and $24^{\circ}$ (freestream conditions: $M=2.85, R e=7.0 \times 10^{7}$ per length unit, $\delta_{0}=0.023 \mathrm{~m}$ ). (- Gas-kinetic scheme (GKS) on finest grid, ( - - -) GKS on medium grid (only shown for $\alpha=$ $\left.24^{\circ}\right),(-\ldots-)$ GKS on coarsest grid (only shown for $\left.\alpha=24^{\circ}\right),(\circ)$ : experimental data from Settles [33]. Fine grid size: $528 \times 136$ 

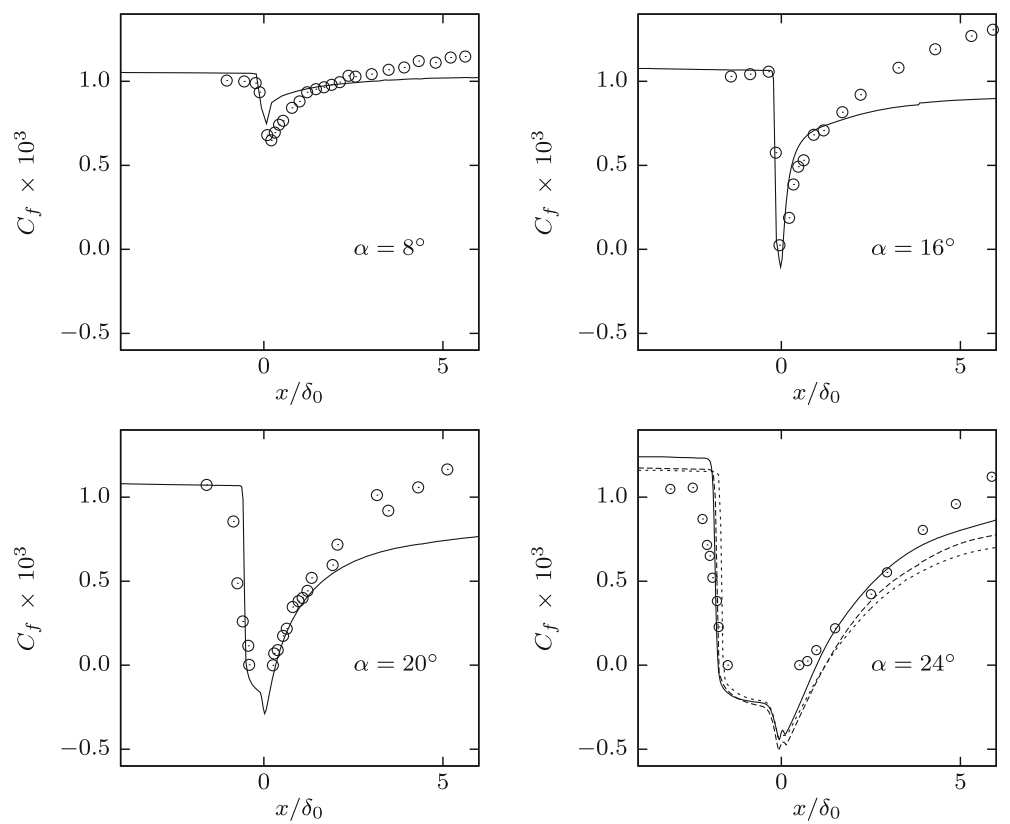

Fig. 3 Skin friction calculated for four different compression corner flows, characterized by angles values of $8^{\circ}, 16^{\circ}, 20^{\circ}$ and $24^{\circ}$ (freestream conditions: $M=2.85, R e=7.0 \times 10^{7}$ per length unit, $\delta_{0}=0.023 \mathrm{~m}$ ). (—) Gas-kinetic scheme (GKS) on finest grid, ( - - - -) GKS on medium grid (only shown for $\alpha=$ $24^{\circ}$ ), ( - - - GKS on coarsest grid (only shown for $\alpha=24^{\circ}$ ), ( o ): experimental data from Settles [33]. Fine grid size: $528 \times 136$

of the flow cases: Fig. 6 refers to the supersonic compression corner investigated by Settles [34], Figs. 10, 14 and 13 refer to the hypersonic cases investigated by Dolling [14], Schuelein [32] and Coleman [11], respectively. Remarkably, this virtual "degree of rarefaction" reaches peak values in the interaction region, which are well beyond the limits
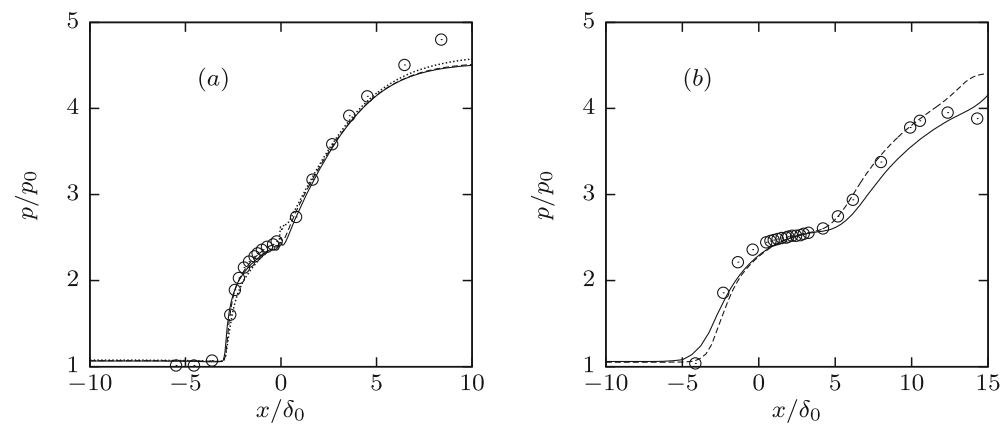

Fig. 4 Distribution of static pressure calculated for the compression corner (a) with an angle of $24^{\circ}$ and for the reflected shock (b) originating form a compression corner at $12^{\circ}$. Freestream conditions (both flows) are $M=2.90, R e_{\theta}=2400$. (— $(-$ GKS on grid 1, ( - - - ) GKS on grid 2, ( ○ ): experimental data from Bookey [5]. Grid sizes compression corner: $384 \times 192,512 \times 168$ respectively, grid size impinging shock: $384 \times 208,496 \times 304$ respectively. Grid 1 and 2 have different resolution and have been generated with different algorithms 

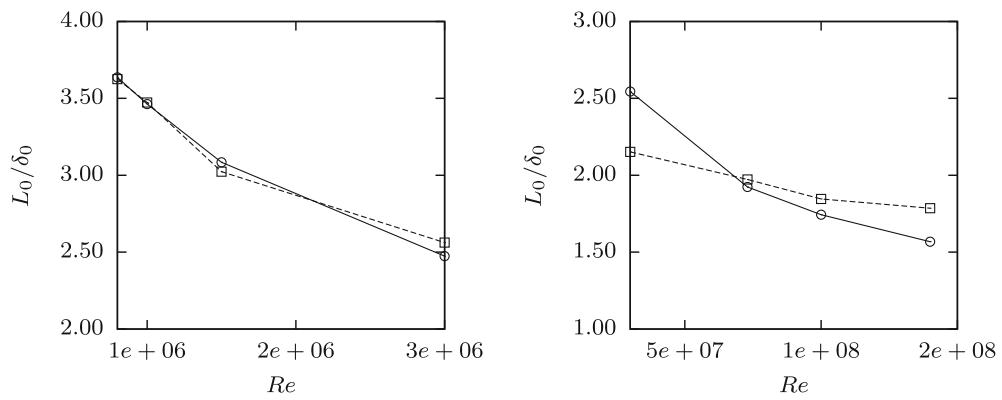

Fig. 5 Interaction length as a function of Reynolds number; the prediction obtained with the gas-kinetic scheme are compared to the empirical law by Settles from [13]. ( - - - - ) present, (__ from Settles

which are conventionally set for continuum regime $(0.01-0.001)$. This finding supports the argument that the gas-kinetic scheme differs from conventional scheme in the interaction region.

\subsection{Transonic flow cases}

\subsubsection{Transonic flow around a RAE 2822 airfoil}

In Case 10 the airfoil is immersed in a flow at Mach $M=0.745$ at an angle of attack $\alpha=3.19^{\circ}$ and Reynolds number $R e_{c}=6300000$ based on airfoil chord. The simulations have been run on conventional, structured C-type grids. Wall resolution has been set in order to solve the laminar sublayer (the centroid of the first computational cell is positioned at a distance $y^{+}<1$ from the wall). The experimental investigation have highlighted an incipient separation of the boundary layer, which fails to be predicted by most linear two-equation turbulence model. This is discussed in various papers for instance in [39]. More sophisticated turbulence models such as algebraic stress models such as the EARSM implementation of the $k-\omega$ or the corrected $k-\omega$ by Wilcox [41] capture the incipient separation correctly. Figure 1 shows the results obtained with the gas-kinetic scheme and the ones obtained with the same solver working with a conventional scheme, based on MUSCL reconstruction and Roe's approximate Riemann solver. Remarkably, the gas-kinetic scheme
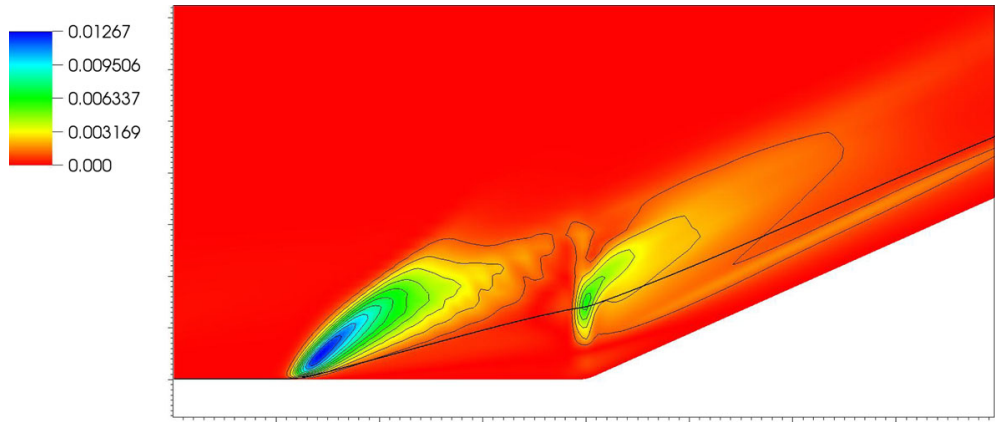

Fig. 6 Compression corner at higher Reynolds number investigated by Settles [34], distribution of the time scales ratio $\epsilon$ 

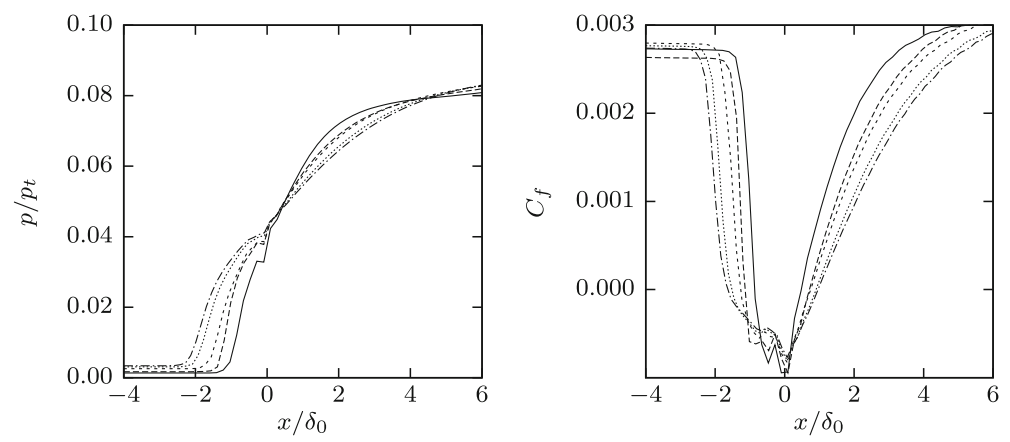

Fig. 7 Compression corner $M=2.9, R_{\delta}=2.2-5.9 \times 10^{4}, \alpha=20^{\circ}$ at various wall temperatures $T_{w} / T_{0}=0.474-0.605-0.775-0.936-1.05$. Static pressure (left) and skin friction coefficient (right). Experimental data from [35]. Results obtained on various grids up to $432 \times 224$. (— $(-\cdots-) T_{w} / T_{0}=0.605,(\cdots \cdots) T_{w} / T_{0}=0.775,(\cdots \cdots) T_{w} / T_{0}=0.936,(-\cdots--) T_{w} / T_{0}=1.05$

provides a rather accurate prediction, whereas the conventional Navier-Stokes scheme places the shock slightly downstream of the position indicted by the measurements.

\subsection{Supersonic compression corner at high and low Reynolds number}

The supersonic flow impinging on a compression corner has been the object of several experimental investigations, such as the ones carried out by Settles [33, 34]. The flow is characterized by a Mach $M=2.85$ and a Reynolds number $\operatorname{Re}_{\theta}=23000$, based on momentum thickness. Four different corners have been used: $8^{\circ}, 16^{\circ}, 20^{\circ}$ and $24^{\circ}$. The $8^{\circ}$ corner does not separate the flow and the $16^{\circ}$ one generates only an incipient separation. The time-averaged separation at $20^{\circ}$ spans about $1 \delta$ and the one at $24^{\circ}$ about $2 \delta$ (where $\delta$ is the incoming boundary layer thickness).

Results from conventional Navier-Stokes scheme are not reported in this paper, as for these flow cases, they tend to become more dependent on numerical details such as reconstruction technique and factorization. The reader is referred to the literature, for instance on the comprehensive review in $[2,16]$ and $[24]$; conventional schemes fail to predict the right
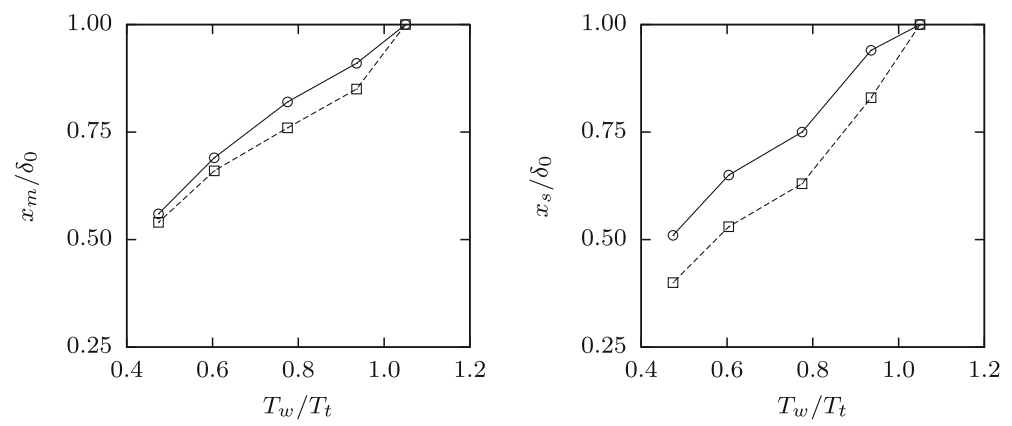

Fig. 8 Effects of wall temperature for a $20^{\circ}$ angle ramp, experimental data from [35]. $T_{w}$ is wall temperature, $T_{t}$ is stagnation temperature, $x_{m}$ is the starting point of interaction from the corner, $x_{s}$ is the starting point of separation from the corner. (— 
Fig. 9 Compression corner $M=4.95$, Experimental values from Dolling et al. [14].

Adiabatic wall conditions.

Results obtained on various grids up to $384 \times 160$

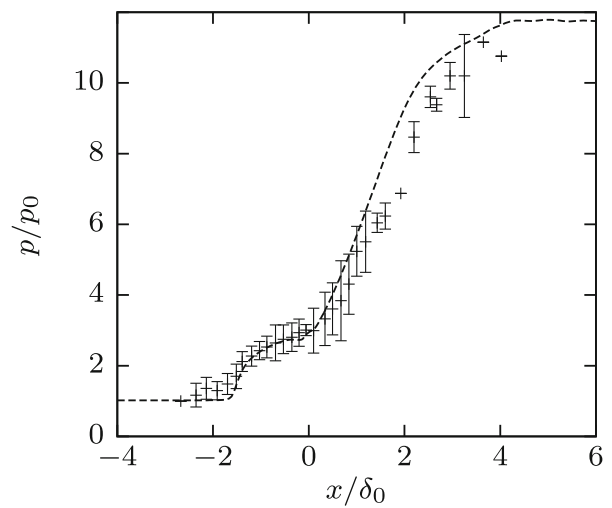

shock position and separation length, the whole interaction region is often translated downstream, the extension of the separation is often underestimated. The turbulent gas-kinetic scheme developed in this study does not provide a "perfect" agreement with experiments but does better than conventional schemes. Figures 2 and 3 show the reasonably good agreement of predictions in all four cases in terms of wall pressure and skin friction coefficient.

In order to generate data for a benchmark case for LES and DNS methods, the same flow, at much a lower Reynolds number of $R e_{\theta}=2400$, has been investigated many years later also in Princeton and published in [5].

The first experiment of this campaign concerns a supersonic compression corner with an angle of $24^{\circ}$. Within the same investigation an shock impinging with an angle of $12^{\circ}$ onto a turbulent boundary layer has also been investigated. The two flow generates a similar shock-boundary layer interaction. Figure 4 shows the pressure distributions for both the compression corner and the impinging shock, which are in reasonably good agreement with the experiments.

For both flow cases, the one investigated by Settles and the one investigated by Bookey, the sensitivity to the Reynolds number has been investigated with the gas-kinetic scheme. Figure 5 shows the interaction length as well as the separation point as a function of the Reynolds number. Both quantities are compared to the values estimated with an empirical
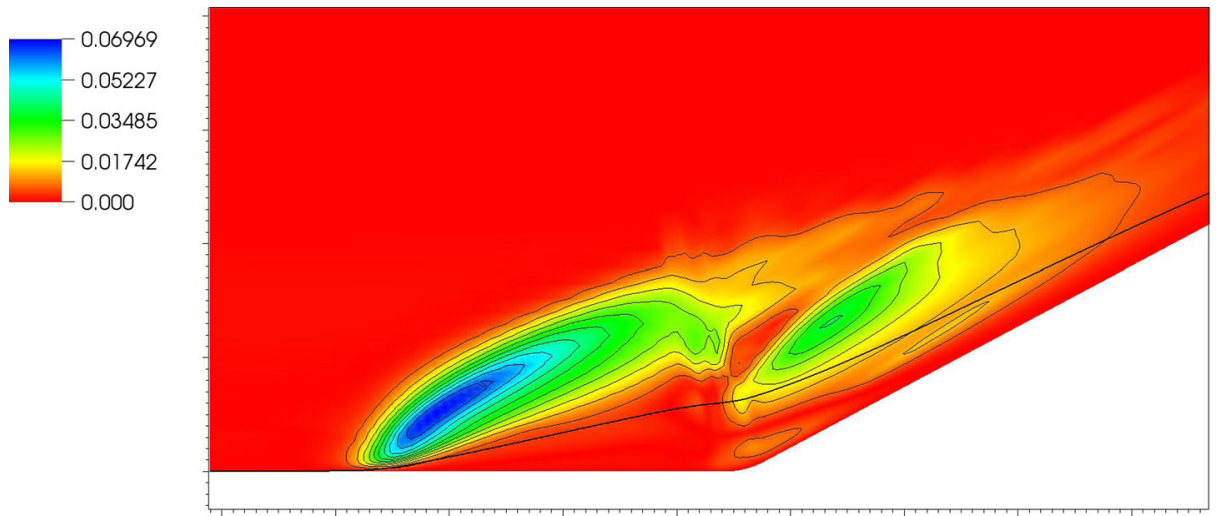

Fig. 10 Compression corner $M=4.95$, Experimental values from Dolling et al. [14]. Adiabatic wall conditions. Results obtained on various grids up to $384 \times 160$ 
law devised by Settles, details can be found in [13]. The prediction is very accurate at lower Reynolds number and barely acceptable in the other case.

The distribution of the time scales ratio $\epsilon$ is shown in Fig. 6 for the case at higher Reynolds. In both cases, it is evident that $\epsilon$ assumes the larger values in the interaction area, that is, where the results obtained with the gas-kinetic scheme and conventional schemes differ most.

\subsubsection{High-enthalpy flows}

The effect of wall temperature and in particular the reduction in separation length caused by the "cold wall" boundary condition is evident from the experiment by Spaid and Frishett [35], who investigated the interaction between shock and the turbulent boundary layer in a compression corner $\left(M=2.9, \operatorname{Re}_{\delta}=2.2-5.9 \times 10^{4}, \alpha=20^{\circ}\right)$ with variable wall temperature. The pressure and skin friction coefficient profiles for all wall temperatures are shown in Fig. 7, the relative changes in interaction length calculated with the gas-kinetic scheme are plotted in Fig. 8 and compared with the experimental data.
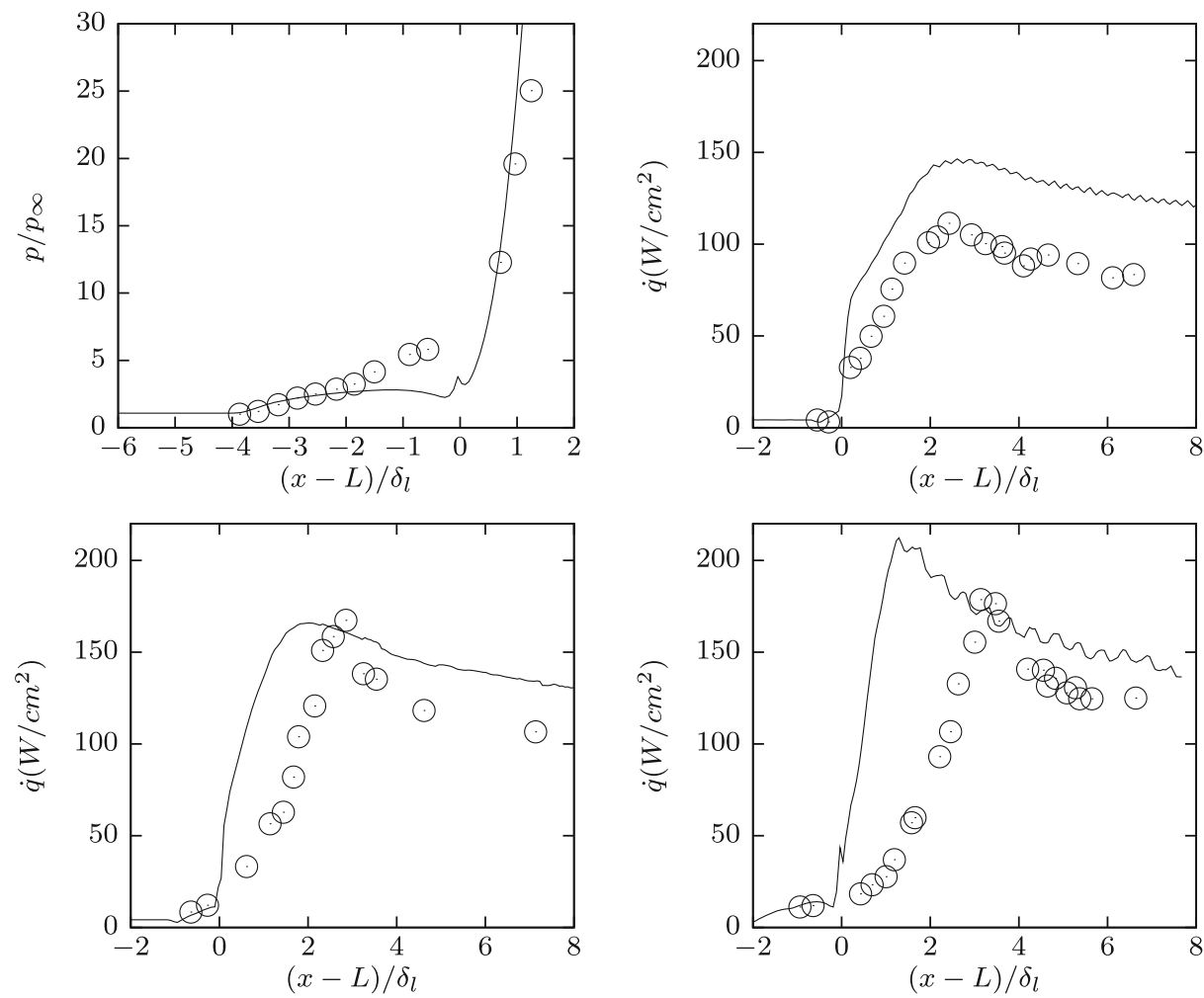

Fig. 11 Compression ramp, $M=9.22, R e=4.8 \times 10^{7}, T_{w} / T_{0}=0.30$, Static pressure for $\alpha=38^{\circ}$ (top, left), heat transfer rate for $\alpha=30^{\circ}, 34^{\circ}$ and $38^{\circ}$, experimental data from [11]. Fine grid size: $1536 \times 80$ 

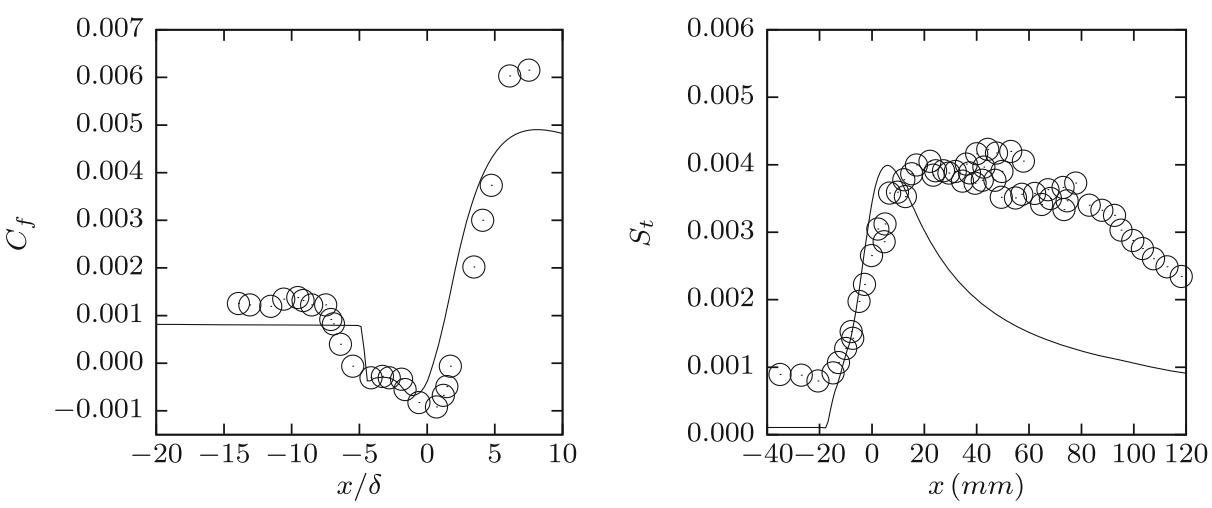

Fig. 12 Reflected shock, $M=5.00, R e=3.5 \times 10^{7}, \alpha=14^{\circ}$, Skin friction, Stanton number, experimental data from [32]. Results obtained on various grids up to $768 \times 288$

\subsection{Hypersonic flows}

\subsubsection{Hypersonic compression corner at Mach 4.95}

This flow case has been investigated by Dolling et al. [14]. Adiabatic wall conditions are assumed. Reynolds number is 877000 based on boundary layer thickness. Results from RANS and hybrid simulations can be found in Edwards et al. [15]. In Fig. 9 the pressure distribution predicted by the turbulent gas-kinetic scheme is compared to the experimental values [14], showing an acceptable agreement. In Fig. 10 the distribution of the time scales ratio $\epsilon$ is shown. It is evident that the highest values arise in the interaction area, that is where the results provided by the gas-kinetic scheme and conventional schemes differ most.
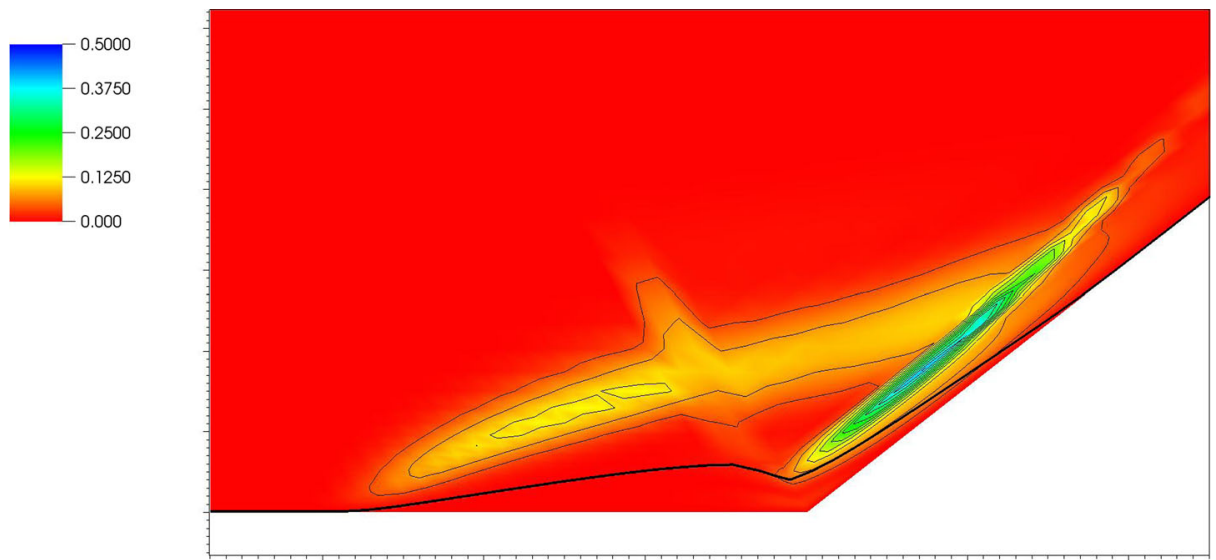

Fig. 13 Compression ramp, $M=9.22, R e=4.8 \times 10^{7}, \alpha=38^{\circ}, T_{w} / T_{0}=0.30$, distribution of $\epsilon$ as in Eq. 21 in the interaction region. Pressure contour lines and the sonic line have been added for reference 

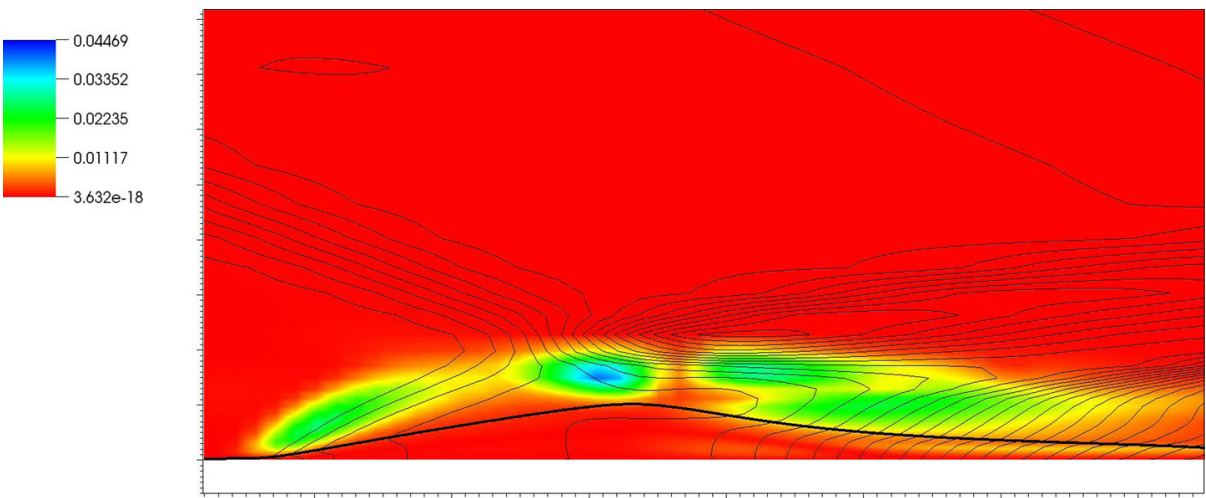

Fig. 14 Reflected shock, $M=5.00, R e=3.5 \times 10^{7}, \alpha=14^{\circ}$, distribution of $\epsilon$ as in Eq. 21 in the interaction region. Pressure contour lines and the sonic line have been added for reference

\subsubsection{High-enthalpy flows}

Two test cases have been so far simulated: the compression ramp at $M=9$ investigated in [11] and the shock - boundary layer caused by an incident oblique shock investigated in [32]. In the first case ramp angles of $30^{\circ}, 34^{\circ}$ and $38^{\circ}$ respectively have been considered. In the second case, only the shock wave generated by a $14^{\circ}$ ramp has been investigated. Both cases are characterized by a "hot" flow, with a $T_{w} / T_{\infty}$ of approximately 0.30 and 0.72 respectively. Figure 11 shows static pressure and heat transfer rate for the compression ramp. Figure 12 shows skin friction coefficient and heat transfer rate profiles for the reflected shock case. The two-dimensional RANS approach clearly misses the effects of three-dimensional vortices and shock unsteadiness, whose relevance tends to increase with Mach. In both case, the agreement with experiments is less good than in the transonic and supersonic flow cases, consistently with other researchers findings, as documented for instance in the reviews by Babinsky [2] and Roy et al. [30]. The heat transfer rate profiles in particular differ evidently from experiments. However, in contrast to the results obtained with conventional schemes, the separation lengths as well as the heat transfer peaks are captured with errors included in a 10-20\% range (for instance in terms of heat transfer peak or position and length of the interaction). Such deviations may in most cases be considered to be within engineering tolerances, that is, of the same order of magnitude of other inaccuracies within a design process. The time scales ratios, shown in Figs. 13 and 14, assume "rarefied" peak values across the shocklayers. This supports once again the assumption that the added-value of gas-kinetic schemes lies with the ability to react to this degree of rarefaction.

\section{Conclusions}

RANS simulations often rely on eddy viscosity to model the effects of unresolved turbulent fluctuations. In special flow conditions, e.g. in the presence of a compression shock, the unresolved scales of motion might not be negligibly smaller than the resolved ones and hence make the diffusion process in conventional schemes not always physically consistent. In the course of the latest decades a number of ad-hoc corrections to standard turbulence 
models have been put forward, precisely to limit the eddy viscosity in the presence of strong gradients.

A different approach is explored in this study: the multiscalar mechanism of gas-kinetic schemes is exploited to perform RANS simulations. The unresolved turbulent scales of motion are accounted for by a turbulent relaxation time, which is obtained from eddy viscosity and a correction to account for local "rarefaction". Eddy viscosity is obtained from a standard $k$ - $\omega$ turbulence model (original version), solved alongside the conservative variables. A number of test cases with strong shock-boundary layer interaction have been tested. Interestingly, the results obtained with the gas-kinetic scheme in this study are more accurate (position and length of the separation, heat transfer rate) than the ones provided by the same turbulence model and a conventional Navier-Stokes scheme (available in the literature). The rationale is put forward that the more accurate predictions are related to the ability of the gas-kinetic scheme to account for a high unresolved-to-resolved scales ratio.

In the future gas-kinetic schemes might be able to improve the simulation of turbulence in a number of ways: (i) additional turbulence models, beside the $k-\omega$, might benefit from the gas-kinetic scheme, (ii) the application to Large Eddy Simulation would also be interesting, as the smallest resolved and the largest unresolved scales of motion are, by definition, close to each other, (iii) the non-linear turbulent stress generated by a secondorder Chapman-Enskog expansion would provide additional accuracy without the need to calibrate additional coefficients as is the case in most higher-order turbulence models.

\section{References}

1. Alder, B.J., Wainwright, T.: Studies in molecular dynamics. i. general method. J. Chem. Phys. 31(2), 459-466 (1959)

2. Babinsky, H., Harvey, J.K.: Shock wave-boundary-layer interactions. Cambridge University Press New York (2011)

3. Bhatnagar, P., Gross, E., Krook, M.: A model for collision processes in gases. I. Small amplitude processes in charged and neutral one-component systems. Phys. Rev. 94(3), 511-525 (1954)

4. Bird, G.A.: Molecular gas dynamics and the direct simulation of gas flows (1994)

5. Bookey, P., Wyckham, C., Smits, A.: Experimental investigations of Mach 3 shock-wave turbulent boundary layer interactions. AIAA Paper No. 2005-4899 (2005)

6. Cercignani, C.: The Boltzmann equation and its applications. Springer, New York (1988)

7. Cercignani, C.: Rarefied gas dynamics: from basic concepts to actual calculations. Cambridge University Pracy, Cambridge (2000)

8. Chen, H., Kandasamy, S., Orszag, S., Shock, R., Succi, S., Yakhot, V.: Extended Boltzmann kinetic equation for turbulent flows. Science 301(5633), 633-636 (2003)

9. Chen, H., Orszag, S., Staroselsky, I., Succi, S.: Expanded analogy between Boltzmann kinetic theory of fluids and turbulence. J. Fluid Mech. 519(1), 301-314 (2004)

10. Chou, S., Baganoff, D.: Kinetic flux-vector splitting for the Navier-Stokes equations. J. Comput. Phys. 130(2), 217-230 (1997)

11. Coleman, G., Stollery, J.: Heat transfer from hypersonic turbulent flow at a wedge compression corner. J. Fluid Mech. 56(04), 741-752 (1972)

12. Cook, P., McDonald, M., Firman, M.: Aerofoil RAE 2822-pressure distributions, and boundary layer andwake measurements. Experimental data base for computer program assessment. AGARD Advisory (1979)

13. Délery, J., Marvin, J., Reshotko, E.: Shock-wave boundary layer interactions. AGARDograph 280 (1986)

14. Dolling, D.S., Erengil, M.E.: Unsteady wave structure near separation in a Mach 5 compression rampinteraction. AIAA J. 29(5), 728-735 (1991)

15. Edwards, J.R.: Numerical simulations of shock/boundary layer interactions using time-dependent modeling techniques: A survey of recent results. Prog. Aerosp. Sci. 44(6), 447-465 (2008)

16. Goldberg, U., Peroomian, O., Chakravarthy, S.: Application of the k-e-R Turbulence Model to WallBounded Compressive Flows. AIAA Paper No. 1998-0323 (1998) 
17. Hellsten, A.K.: New advanced kw turbulence model for high-lift aerodynamics. AIAA J. 43(9), 18571869 (2005)

18. Li, Q., Fu, S., Xu, K.: Application of gas-kinetic scheme with kinetic boundary conditions in hypersonic flow. AIAA J. 43(10), 2170-2176 (2005)

19. Li, Q., Xu, K., Fu, S.: A high-order gas-kinetic Navier-Stokes flow solver. J. Comput. Phys. 229(19), 6715-6731 (2010)

20. Liao, W., Luo, L., Xu, K.: Gas-kinetic scheme for continuum and near-continuum hypersonic flows. J. Spacecr. Rocket. 44(6), 1232-1240 (2007)

21. Mandal, J., Deshpande, S.: Kinetic flux vector splitting for Euler equations. Comput. Fluids 23(2), 447478 (1994)

22. May, G., Srinivasan, B.: An improved gas-kinetic BGK finite-volume method for three-dimensional transonic flow. J. Comput. Phys. 220(2), 856-878 (2007)

23. Menter, F.R.: Improved two-equation k-omega turbulence models for aerodynamic flows. NASA STI/Recon. Tech. Report N 93(22), 809 (1992)

24. Menter, F.R., Rumsey, C.L.: Assessment of two-equation turbulence models for transonic flows. American Institute of Aeronautics and Astronautics (1994)

25. Nordsieck, A., Hicks, B.: Monte carlo evaluation of the boltzmann collision integral. In: Proceedings of 5th International Symposium on Rarefied Gas Dynamics, pp. 695-710. Academic Press (1967)

26. Ohwada, T., Xu, K.: The kinetic scheme for the full-Burnett equations. J. Comput. Phys. 201(1), 315332 (2004)

27. Rahman, A.: Correlations in the motion of atoms in liquid argon. Phys. Rev. 136(2A), A405 (1964)

28. Righi, M.: A finite-volume gas-kinetic method for the solution of the Navier-Stokes equations, vol. (117) (2013)

29. Righi, M.: A modified gas-kinetic scheme for turbulent flow. Commun. Comput. Phys. 16(1), 239-263 (2014)

30. Roy, C.J., Blottner, F.G.: Review and assessment of turbulence models for hypersonic flows. Progress Aerosp. Sci. 42(7), 469-530 (2006)

31. Rubinstein, R., Barton, J.M.: Nonlinear reynolds stress models and the renormalization group. Phys. Fluids A: Fluid Dyn. (1989-1993) 2(8), 1472-1476 (1990)

32. Schülein, E.: Skin friction and heat flux measurements in shock/boundary layer interaction flows. AIAA J. 44(8), 1732-1741 (2006)

33. Settles, G., Fitzpatrick, T., Bogdonoff, S.: Detailed study of attached and separated compression corner flowfields in high Reynolds number supersonic flow. AIAA J. 17(6), 579-585 (1979)

34. Settles, G., Vas, I., Bogdonoff, S.: Details of a shock-separated turbulent boundary layer at a compression corner. AIAA J. 14(12), 1709-1715 (1976)

35. Spaid, F.W., Frishett, J.C.: Incipient separation of a supersonic, turbulent boundary layer, including effects of heat transfer. AIAA J. 10(7), 915-922 (1972)

36. Speziale, C.G.: On nonlinear kl and k- $\varepsilon$ models of turbulence. J. Fluid Mech. 178, 459-475 (1987)

37. Tcheremissine, F.G.: A method for direct numerical integration of boltzmann equation. In: Numerical Method in the Theory of Gases, pp. 43-63. USSR Academy Science (1969)

38. Wallin, S., Johansson, A.: A new explicit algebraic Reynolds stress turbulence model for 3 D flow. In: Symposium on Turbulent Shear Flows, 11 th, pp. 13-13, Grenoble, France (1997)

39. Wallin, S., Johansson, A.: An explicit algebraic Reynolds stress model for incompressible and compressible turbulent flows. J. Fluid Mech. 403, 89-132 (2000)

40. Wilcox, D.C. Turbulence Modeling for CFD, 3rd. DCW Industries, Inc., La Canada CA (2006)

41. Wilcox, D.C.: Formulation of the kw turbulence model revisited. AIAA J. 46(11), 2823-2838 (2008)

42. $\mathrm{Xu}, \mathrm{K}$.: Gas-kinetic schemes for unsteady compressible flow simulations. VKI, Computational Fluid Dynamics, Annual Lecture Series, 29th. Rhode-Saint-Genese, Belgium (1998)

43. $\mathrm{Xu}, \mathrm{K}$. : A gas-kinetic BGK scheme for the Navier-Stokes equations and its connection with artificial dissipation and Godunov method. J. Comput. Phys. 171(1), 289-335 (2001)

44. Xu, K., He, X., Cai, C.: Multiple temperature kinetic model and gas-kinetic method for hypersonic non-equilibrium flow computations. J. Comput. Phys. 227(14), 6779-6794 (2008)

45. $\mathrm{Xu}, \mathrm{K}$., Huang, J.: A unified gas-kinetic scheme for continuum and rarefied flows. J. Comput. Phys. 229(20), 7747-7764 (2010)

46. Xu, K., Mao, M., Tang, L.: A multidimensional gas-kinetic BGK scheme for hypersonic viscous flow. J. Comput. Phys. 203(2), 405-421 (2005)

47. $\mathrm{Xu}, \mathrm{K}$. , Prendergast, K.: Numerical Navier-Stokes solutions from gas kinetic theory. J. Comput. Phys. 114(1), 9-17 (1994) 
48. Xuan, L., Xu, K.: A new gas-kinetic scheme based on analytical solutions of the BGK equation. Journal Computer Physics (2012)

49. Yen, S.: Numerical solution of the nonlinear boltzmann equation for nonequilibrium gas flow problems. Ann. Rev. Fluid Mech. 16(1), 67-97 (1984)

50. Yoshizawa, A.: Statistical modeling of a transport equation for the kinetic energy dissipation rate. Phys. Fluids 30, 628-631 (1987) 